\title{
Neutrinos and the synthesis of heavy elements: the role of gravity
}

\author{
O. L. Caballero ${ }^{1,2,3, a}$, R. Surman ${ }^{4}$, and G. C. McLaughlin ${ }^{5}$ \\ ${ }^{1}$ Department of Physics, University of Guelph, Guelph, ON, Canada \\ ${ }^{2}$ ExtreMe Matter Institute EMMI, GSI Helmholtzzentrum für Schwerionenforschung GmbH, 64291 Darmstadt, Germany \\ ${ }^{3}$ Institut für Kernphysik, Technische Universität Darmstadt, 64289 Darmstadt, Germany \\ ${ }^{4}$ Department of Physics, University of Notre Dame, Notre Dame, IN, USA \\ ${ }^{5}$ Department of Physics, North Carolina State University, Raleigh, NC, USA
}

\begin{abstract}
The synthesis of heavy elements in the Universe presents several challenges. From one side the astrophysical site is still undetermined and on other hand the input from nuclear physics requires the knowledge of properties of exotic nuclei, some of them perhaps accessible in ion beam facilities. Black hole accretion disks have been proposed as possible r-process sites. Analogously to Supernovae these objects emit huge amounts of neutrinos. We discuss the neutrino emission from black hole accretion disks. In particular we show the influence that the black hole strong gravitational field has on changing the electron fraction relevant to the synthesis of elements.
\end{abstract}

\section{Introduction}

Where and how heavy elements are produced in the Universe is one of the fundamental questions in science. Attempting to answer it requieres efforts from different fields. From one side, astronomical observations provide information about the abundances of heavy elements, while nuclear physics brings insights on the details of the reactions taking place among nuclei and the resulting final abundances. The goal is to be able to emulate the thermodynamical conditions of some stellar site, to evolve a reaction network under those conditions, and finally to reproduce the observed abundaces. However, at this point, there is still controversy on the astronomical site(s), and the nuclear properties of many of the nuclei participating in the reactions are uncertain. New experimental facilities bring hope in the study of nuclei far from stability (e.g. [1$3])$. These studies will also shed light on theoretical models, needed to determine the properties of perhaps never accesible nuclei.

Among all these different ingredients, neutrinos play a crucial role. Via weak interactions they can drive a medium proton-rich or neutron-rich. This together with the thermodynamical evolution of the matter determine what kind of elements are synthetized.

The flux of neutrinos emitted from stellar sources such as supernovae and black hole accretion disks (two of the suggested sites of r-process nucleosynthesis), can be affected by different kind of physics, e.g flavor oscillations $[4,5]$ and coherent scattering $[6,7]$. In previous works $[8,9]$, we have studied the influence that gravity has on the emission of neutrinos and the production of heavy el-

\footnotetext{
ae-mail: 1caballe@umail.iu.edu
}

ements in outflows emerging from black hole accretion disks. Although our studies have been focused on these particular sites, the effects of strong gravitational fields on neutrino emission are important in any other enviroment where neutrinos are copiously produced in the vecinity of massive central object. Of particular importance is the consideration of the 3D geometry of the source, as the relativistic effects depend on the space-time curvature. Below we discuss some more details of the effect of general relativity on neutrino fluxes and the synthesis of heavy elements in black hole accretion disks.

\section{General Relativistic Effects on Neutrino Fluxes}

The strong gravitational field generated by a compact object changes the geometry of the space-time around it. This affects the flux of neutrinos observed at a certain distance from the central object. The main effects of the gravitational field on the fluxes are the shifts of energies and the deformation of the solid angle that the source subtends as seen by the observer $d \Omega_{o b}$. The latter can be determined via the deflection of the neutrino trajectories and requieres to find their null geodesics in a given curvature. The emitted energy $E_{e m}$ and the observed energy $E_{o b}$ are related by $E_{e m}=\left(1+z^{*}\right) E_{o b}$ where $\left(1+z^{*}\right)$ is the redshift.

The effective neutrino flux observed at some distance $r_{o b}$ from the compact object is

$$
\phi^{e f f}=\frac{1}{4 \pi} \int d \Omega_{o b} \times \phi_{o b}\left(E_{o b}\right) .
$$

The starting point to perform a transformation from the fluxes in a flat geometry (here we call them Newtonian 
fluxes) to the ones in a curved one (General Relativistic fluxes) is the conservation of the number density in phase space [15]. This leads to write the observed general relativistic fluxes as

$$
\phi^{e f f} \propto \frac{1}{4 \pi} \int d \Omega_{o b} \times \frac{E_{o b}^{2}}{\exp \left(E_{o b}\left(1+z^{*}\right) / T_{e m}\right)+1},
$$

where we have written the neutrino Fermi-Dirac distribution in terms of the temperature at the emission point $T_{e m}$ (usually the known amount from numerical simulations). Both the redshift $1+z^{*}$ and and the solid angle $d \Omega_{o b}$ depend on the space-time geometry, and therefore on the details of the matter distribution of the compact object.

\section{Reaction rates}

A key factor in determining the type of nuclear products synthetized in an astrophyscial site (particularly in neutrino-driven like environments) is the proton to neutron fraction or electron fraction $Y_{e}$. If $Y_{e}>0.5$ the medium is proton-rich while if $Y_{e}<0.5$ it is neutron-rich. In this kind of environments the initial thermodynamical conditions of the matter sorrunding the compact object are such that it is dissociated into electron, protons and neutrons. Then, the main reactions setting the matter composition are:

$$
\begin{aligned}
e^{+}+n \leftrightarrow p+\bar{v}_{e}, \\
e^{-}+p \leftrightarrow n+v_{e} .
\end{aligned}
$$

If the flux of electron neutrinos is larger than the electron antineutrinos the inverse reaction of equation 4 will drive the matter proton rich. Conversely, if the electron antineutrino flux is larger than the neutrino flux the matter will be neutron rich. On the other side, if both neutrino and antineutrino fluxes are weak the forward reactions, electron capture on protons and positron captures on neutrons, will play a more important role than neutrinos in determining the electron fraction.

The electron fraction $\tilde{Y}_{e}$ that is obtained by taking into account only the reverse reactions of eqs. 3 and 4 depends on the absoption rates of these processes. $\tilde{Y}_{e}$ depends on the neutrino fluxes and is therefore also affected by the strong gravitational field. In terms of the observed fluxes $\phi^{\text {eff }}$ (eq. 2) the rate of absorption of neutrinos on neutrons is

$$
\lambda_{v_{e} n}=b \int_{0}^{\mathrm{inf}} \phi_{v_{e}}^{e f f}\left(E_{o b}+\Delta\right)^{2} \sqrt{1-\frac{m_{e}^{2}}{\left(E_{o b}+\Delta\right)^{2}}} W_{M} d E_{o b}
$$

and the rate of absorption of antineutrinos on protons is

$$
\lambda_{\bar{v}_{e} p}=b \int_{\Delta+m_{e}}^{\mathrm{inf}} \phi_{\bar{v}_{e}}^{e f f}\left(E_{o b}-\Delta\right)^{2} \sqrt{1-\frac{m_{e}^{2}}{\left(E_{o b}-\Delta\right)^{2}}} W_{\bar{M}} d E_{o b},
$$

where $W_{M}=1+1.1 E_{o b} / m_{n}$ and $W_{\bar{M}}=1-7.1 E_{o b} / m_{n}$ are the weak magnetism corrections [10], $m_{n}, m_{e}$ are the neutron and electron masses respectively, $\Delta$ is the neutronproton mass difference, and $b=9.704 \times 10^{-50} \mathrm{~cm}^{2} \mathrm{keV}^{-2}$.

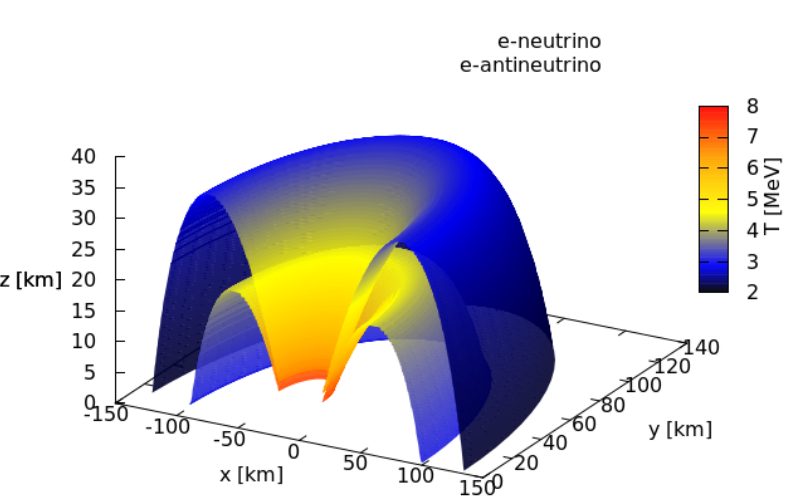

Figure 1. Electron neutrino (outter) and antineutrino (inner) surfaces corresponding to a snapshop at $\mathrm{t}=20 \mathrm{~ms}$, of a hydrodynamical simulation of a torus around a 3 solar mass black hole.

\section{Astrophysical site: Accretion disk outflows}

A possible scenario after the merger of two compact objects (black hole-neutron star or neutron star-neutron star) is the formation of an accretion disk or torus around a black hole. Given the inital conditions of the progenitors the matter of the disk is neutron rich and hot enough to be dissociated in nucleons. Some fraction of this matter can be ejected in hot outflows, presenting an interesting scenario for the synthesis of neutron-rich elements.

The results presented here and in ref. [8] are based on a time depedent hydrodynamical simulation of accreting matter around a 3 solar masses black hole with a spin parameter $a=0.8$ (for more details on the simulation see [11]). The initial disk conditions emulate those of mergers. The initial electron fraction $Y_{e}$ is 0.1 as suggested by merger simulations [11]. The disk evolves from $t=0 \mathrm{~s}$ when the Shakura and Sunyaev alpha viscosity terms are turned on. In this astrophysical scenario neutrinos are copiously emitted. The emission points correspond to the neutrino surfaces, the places where after being trapped by the high density conditions, neutrinos can freely travel. Figure 1 shows a transversal cut of the electron neutrino and antineutrino surfaces for this disk model at $t=20$ ms. The $z$ axis correspond to the actual decoupling height, and the colored scale shows the neutrino temperature $T_{e m}$, which is crucial in calculating the neutrino fluxes as described in eq. 2 . The reactions and details used to calculate these surfaces are discussed in ref. [12]. The differences in the neutrino decoupling surfaces for each flavor (some caused by gravity) has important consequences on the nucleosynthetic outcome.

For the outflow we adopted standard neutrino-driven wind trajectories parameterized in entropy and timescale or acceleration. See for example ref. [13] for supernova and ref. [14] for accretion disk outflows. Our outflow follows a radial streamline that starts at $r=30 \mathrm{~km}$ from the black hole and extends to thousands of kilometers away. 


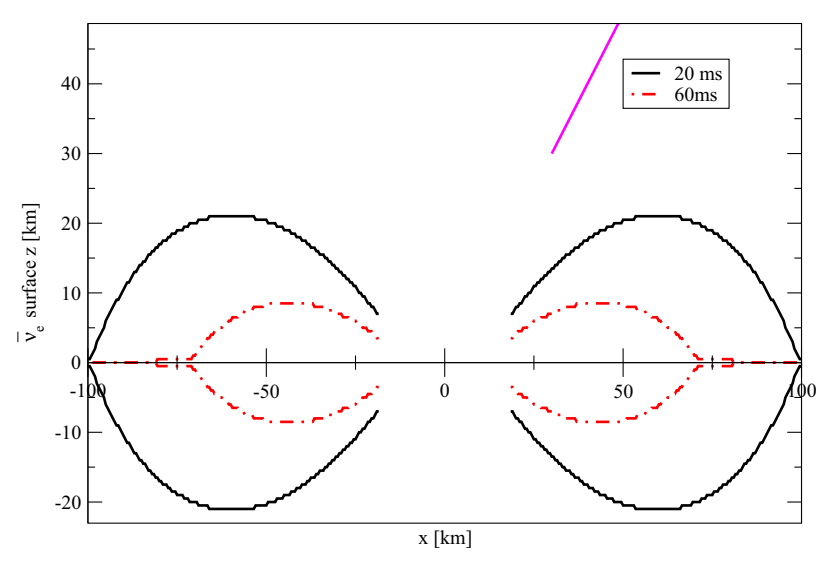

Figure 2. A transversal cut of the electron antineutrino surfaces at two different times, $\mathrm{t}=20$ and $60 \mathrm{~ms}$. The magenta line shows the outflow trajectory starting at $x=30 \mathrm{~km}, \mathrm{z}=30 \mathrm{~km}$, It is from this point that we launch the outflow and follow the evolution of the neutrino reaction rates.

Figure 2 shows the electron antineutrino surfaces at two different times $\mathrm{t}=20$ and $60 \mathrm{~ms}$, and a segment of the outflow trajectory (magenta line). As time passes and material is dragged into the black hole the neutrino surfaces shrink.

\subsection{Neutrino fluxes}

We calculated the neutrino fluxes for this astrophysical environment, as in eq. 2 . The observers are the points of the outflow trajectory where the reactions of eqs. 3 and 4 take place. The emitters are the points on the neutrino surfaces.

For each point in the outflow we found the null geodesics that connect them to each point on the neutrino surface. This procedure determines the solid angles $d \Omega_{o b}$. The redshift $1+z^{*}$ also depends on the position of the outflow trajectory $r_{o b}$ and on the emission points on the neutrino surface $r_{e m}$. We calculated both redshifts and null geodesics in the Schwarzschild metric in a similar way as in ref. [9].

At $t=20 \mathrm{~ms}$ electron antineutrinos are hotter (see colored scale in figure 1) and their fluxes are larger than electron neutrino fluxes. This is true regardless of the spacetime curvature, as can been seen when we compare the fluxes by flavor in figure 3 (black vs red lines), where we have plotted the fluxes seen at $z_{o b}=x_{o b}=100 \mathrm{~km}$. This difference results in more antineutrino captures on protons, driving the material neutron rich. When general relativistic effects are included the more energetic antineutrinos that are emitted closer to the black hole are more redshifted than the neutrinos. This causes the large energy tails of the fluxes to be reduced (compare dotteddashed lines for Newtonian (N) neutrinos with solid lines for the relativistic ones (GR) in figure 3). As a result in a curved space-time the electron antineutrinos capture rates are more reduced when compared to the Newtonian rates and the material becomes less neutron rich. As an example for an outflow trajectory with an entropy per baryon of 30 , the Newtonian electron fraction $\tilde{Y}_{e}$ tends to 0.47 near $z_{o b}=x_{o b}=100 \mathrm{~km}$, while the relativistic is $\tilde{Y}_{e}=0.49$ (for

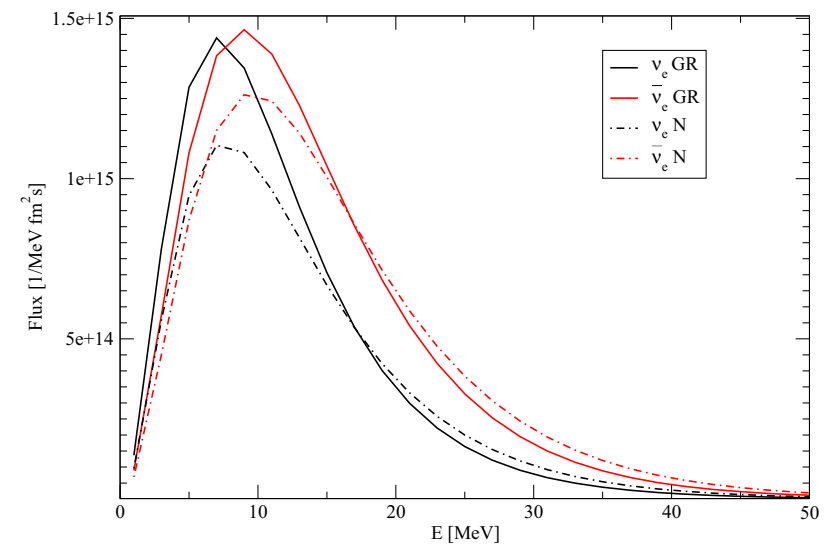

Figure 3. Electron neutrino (black) and antineutrino (red) fluxes as registered at $20 \mathrm{~ms}$ at a point located at $x_{o b}=z_{o b}=100 \mathrm{~km}$ from the center of the black hole. The solid lines correspond to a general relativistic calculation of the fluxes while the dotteddashed lines describe fluxes in a Newtownian calculation.

an illustration of the behavior of electron fraction, with a specific outflow, with and without general relativity see figure 2 of ref. [8]).

Note however, that the dynamical evolution changes the emission points as is shown in figure 2. Then the time dependence of the neutrino surfaces plays an important role. Figure 4 shows the neutrino fluxes at two different times $t=20 \mathrm{~ms}$ (red lines) and $t=60 \mathrm{~ms}$ (blue lines) as seen by an observer located at $x_{o b}=z_{o b}=100 \mathrm{~km}$ from the black hole. At $t=20 \mathrm{~ms}$ electron antineutrino fluxes are larger than the electron neutrino fluxes, as it was dicussed above (compare solid vs dashed red lines). However, we see the opposite behavior at $t=60 \mathrm{~ms}$ (see blue lines in figure 4): the electron neutrino fluxes are larger. This is because as time passes more material has been dragged into the black hole, and althought both surfaces shrink, the electron antineutrino one is much more reduced. As a result at $t=60 \mathrm{~ms}$, neutrino capture on neutrons (eq. 4) dominates and the material becomes proton rich. When general relativity is taken into account the reduction of the antineutrino fluxes is stronger because the antineutrinos are even more redshifted than neutrinos. This makes the medium even more proton rich. In the example mentioned above this translates into a Newtonian $\tilde{Y}_{e} \approx 0.53$, while with GR $\tilde{Y}_{e} \approx 0.56$ (this can be seen in the magenta lines of figure in ref [8]).

\section{Production of ${ }^{56} \mathrm{Ni}$}

The dynamical study of the final abundances requires the knowledge of the electron fraction as a function of time. So far, we have shown the influence of gravity on the electron fraction at two different times, $t=20$ and $t=60$ ms. A detailed calculation of the electron fraction for all times would require repeating the steps described above for small time steps in this interval. This would include finding the neutrino surfaces and the null geodesics at all times and for every point of the outflow trajectory. This procedure would be computationally expensive. Instead 


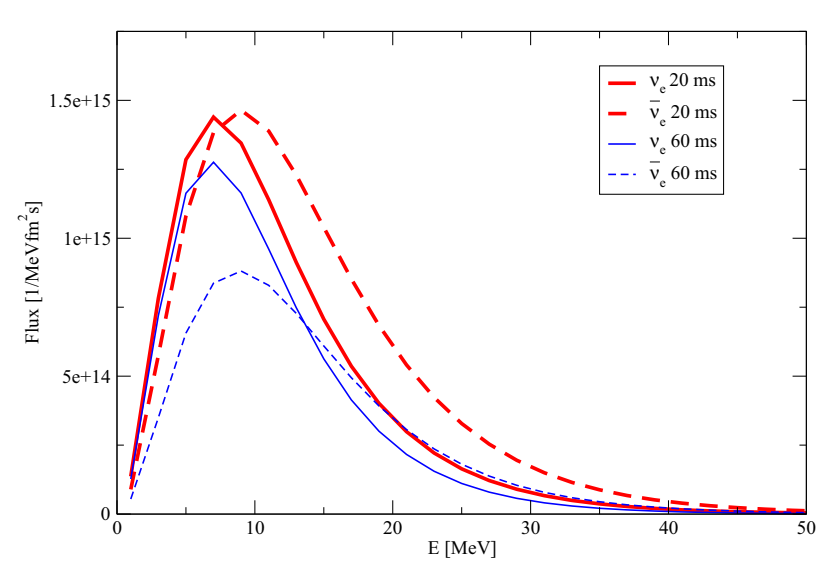

Figure 4. Comparison of electron neutrino (solid) and antineutrino (dashed) fluxes registered at $x_{o b}=z_{o b}=100 \mathrm{~km}$. Red lines correspond to fluxes at $\mathrm{t}=20 \mathrm{~ms}$ and blue lines to $\mathrm{t}=60 \mathrm{~ms}$.

we can think of a simple model. It is natural to expect that the electron fraction would lie in between the limiting values obtained at the two snapshops $t=20$ and $t=60 \mathrm{~ms}$ mentioned above $\tilde{Y}_{e}=0.49$ and $\tilde{Y}_{e}=0.56$. In ref [8] we proposed at linear time dependency of the reaction rates to emulate this time evolution.

In our nucleosynthesis calculations in ref [8] we also sampled the outflow parameter space, allowing a wide variety of thermodynamical outflow conditions. Entropies per baryon $s / k$ were allowed to take values from 20 to 80 , and the effective dynamic time scale $\tau$ was varied beteween 10 and $100 \mathrm{~ms}$. A description of the nuclear reaction network can be found in refs. [16-18]. Under the conditions described above we performed nucleosythesis calculations to find the abundances produced in these outflows. In figure 5 we show the final mass fraction $X(A)$ vs mass number $A$, for an outflow trajectory with entropy per baryon $s / k=30$ and dynamic timescale $\tau=20 \mathrm{~ms}$. The red lines show the GR mass fractions while the black lines correspond to a Newtonian calculation. The enhacement in the production of ${ }^{56} \mathrm{Ni}$ in the GR mass fractions is the result of the larger electron fraction for this case.

For the majority of the outflow conditions we found proton-rich nuclei, with ${ }^{56} \mathrm{Ni}$ the most abundant. This is a direct consequence of the time evolution of the neutrino fluxes. The GR corrections further enhance the protonrichness of the outflow, by increasing the electron fraction. Note however, that for higher entropies, the simulations with GR corrections would lead to $Y_{e}$ above the optimum range for a large ${ }^{56} \mathrm{Ni}$ production. Therefore the mass fraction of ${ }^{56} \mathrm{Ni}$ would be larger in the Newtonian case (see [8] for details on the ${ }^{56} \mathrm{Ni}$ abundance fractions as a function of the outflow condtions).

\section{Conclusions}

The flux of neutrinos observed from a source with a massive central object is significantly different from the flux emitted from the same source in a graviational field free space. This difference affects the neutrino absorption rates

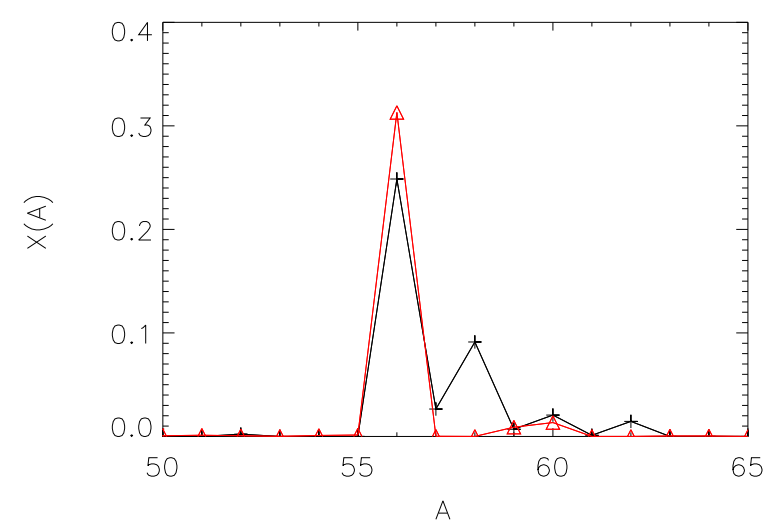

Figure 5. Final mass fractions for an outflow trajectory with $s / k=30$ and $\tau=20 \mathrm{~ms}$, corresponding to a calculation with (red) and without (black) general relativistic corrections.

on nucleons and therefore the electron fraction of the medium. The changes introduced in the electron fraction by the gravitaional field are important enough to alter the nucleosynthesis final abundances.

Due to the initially low electron fraction of their progenitors, merger-type accretion disks have been considered to be good candidates for the synthesis of heavy neutron rich nuclei. We studied the synthesis of elements that occur in black hole accretion disk outflows. Our disk model is based on a hydrodynamical simulation, and the outflow model is similar to standard supernova neutrino winds. We found that gravity plays an important role in setting the electron fraction via its influence on the behavior of neutrinos. The over all change in the neutrino fluxes is a reduction of the high energy tails due to redshifts. This effect is stronger in the electron antineutrino channel, driving the material proton-rich. We found that time evolution plays an important role as the neutrino surfaces shrink when matter is dragged into the black hole. This reduction in the neutrino surfaces combined with the stronger redshifts leads to even more proton rich material and the synthesis of larger amounts of ${ }^{56} \mathrm{Ni}$ for a wide range of outflow conditions.

\section{Acknowledgements}

This work was partially supported by the Natural Sciences and Engineering Research Council of Canada (NSERC)(OLC), the Helmholtz Alliance Program of the Helmholtz Association, contract HA216/EMMI "Extremes of Density and Temperature: Cosmic Matter in the Laboratory" (OLC), and the Department of Energy under contracts DE-FG02- 05ER41398 (RS) and DEFG02-02ER41216 (GCM).

\section{References}

[1] A.B Balantekin et al, Mod. Phys. Lett. A 29, 1430010 (2014) 
[2] H. Gutbrod et al.(Eds) FAIR Baseline Technical Report, ISBN: 3-9811298 0 (2006)

[3] Y. Yano, Nucl. Instrum. Methods Phys. Res. B 261, 1009 (2007)

[4] A. Malkus, J. P. Kneller, G. C. McLaughlin and R. Surman, Phys. Rev. D 86, 085015 (2012)

[5] E. Pllumbi, I. Tamborra, S. Wanajo, H. -Th. Janka, L. Huedepoh, arXiv:1406.2596

[6] C. Horowitz et al. Phys. Rev. C 69, 045804 (2004)

[7] H. Sonoda et al. Phys. Rev. C 75, 042801 (2007)

[8] R.Surman, O. L. Caballero, G. C. McLaughlin, O. Just, H.-Th. Janka, J. Phys. G: Nucl. Part. Phys. 41, 044006 (2014)

[9] O. L. Caballero, G. C. McLaughlin and R. Surman, Astrophys. J. 745, 170 (2012)

[10] C. J. Horowitz, Phys. Rev. D 65, 043001 (2002)
[11] O. Just et al., submitted to MNRAS (2014); arXiv:1406.2687

[12] O. L. Caballero, G. C. McLaughlin, and R. Surman, Phys. Rev. D 80, 123004 (2009)

[13] Y. Z. Qian, et al. Astrophys. J. 471, 331 (1996)

[14] R. Surman and G. C. McLaughlin, Astrophys. J. 618, 397 (2004)

[15] K. S. Thorne, in General Relativity and Cosmology, ed. R. K. Sachs, (New York \& London:Academic Press, 1971), 237

[16] R. Surman, G. C. McLaughlin and N. Sabbatino, Astrophys. J. 743, 155 (2011)

[17] W. R. Hix and F.-K. Thielemann, J. Comput. Appl. Math. 109, 321 (1999)

[18] G. C. McLaughlin, G. M. Fuller and J. R. Wilson, Astrophys. J. 472, 440 (1996) 
